\title{
An unclear, chronic nasal ulcer
}

\author{
Carolina Talhari, M.D. ${ }^{1}$, José Antônio Pedro Mendes Schettini, M.D. ${ }^{2}$, \\ Alexandra Maria Giovanna Brunasso, M.D. ${ }^{3,4}$,Sinesio Talhari, M.D. ${ }^{1}$, Cesare Massone, M.D. ${ }^{5}$ \\ ${ }^{1}$ Department of Dermatology, Institute of Tropical Medicine, Manaus, Brazil \\ ${ }^{2}$ Foundation Alfredo of Matta, Manaus, Brazil \\ ${ }^{3}$ Division of Environmental Dermatology and Venereology, Medical University of Graz, Graz, Austria \\ ${ }^{4}$ Department of Dermatology, Galliera Hospital, Genoa, Italy \\ ${ }^{5}$ Division of General Dermatology, Medical University of Graz, Graz, Austria
}

Key words: mucocutaneous leishmaniasis, nasal ulcer, gingival hyperplasia

Citation: Talhari C, Schettini JA, Brunasso AM, Talhari S, Massone C. An unclear, chronic ulcer. Dermatol Pract Concept 2011;1(1):5. http://dx.doi.org/10.5826/dpc.0101a05.

Editor: Harald Kittler, M.D.

Received: June 5, 2011; Accepted: July 1, 2011; Published: October 31, 2011

Copyright: (02011 Talhari et al. This is an open-access article distributed under the terms of the Creative Commons Attribution License, which permits unrestricted use, distribution, and reproduction in any medium, provided the original author and source are credited.

Funding: None.

Competing interests: The authors have no conflicts of interest to disclose.

All authors have contributed significantly to this publication.

Corresponding author: Cesare Massone, MD, Department of Dermatology, Medical University of Graz, Auenbruggerplatz 8, A-8036 Graz, Austria. Tel.+43.316.385.3235, Fax: +43.316.385.4957. Email: cesare.massone@klinikum-graz.at.

\section{Case report}

A 44-year-old Brazilian man presented with an eight-month history of runny nose, hoarseness and progressive gingival swelling, which interfered greatly with nourishment and oral hygiene. The nasal lesion had appeared three years previous to his visit, while the gingival lesions had been present for a few months. The patient had been already seen by general practitioners and dentists and had received nonspecific therapy. He was not taking any drugs. Physical examination showed a painless ulceration on the right nasal fossa (Figure 1) and hyperplastic erythematous lesions on the upper gingival mucosa (Figure 2). He had no fever and there was no lymphadenopathy nor hepatosplenomegaly. Full blood count, liver and kidney function tests were within the normal range. HIV-serology was negative. Cultures for bacteria, fungus and mycobacteria were negative. Histological examination of a gingival lesion revealed a granulomatous inflammation without necrosis. Fite, Grocott methanamine silver and Periodic Acid Schiff stains were negative.

What is your diagnosis?

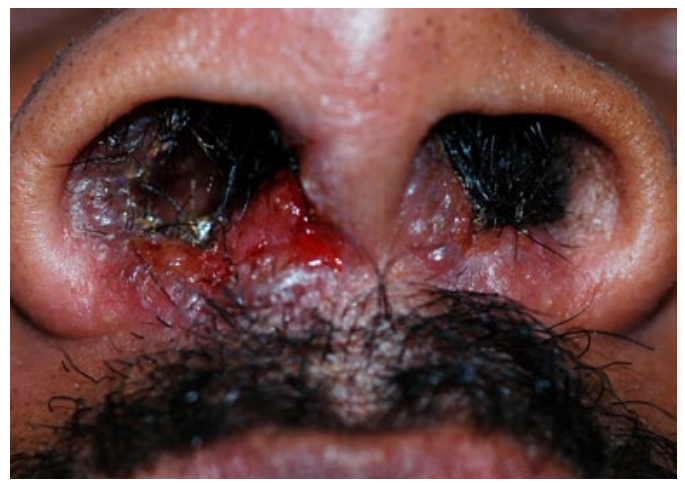

Figure 1. Painless ulceration on the right nasal fossa.

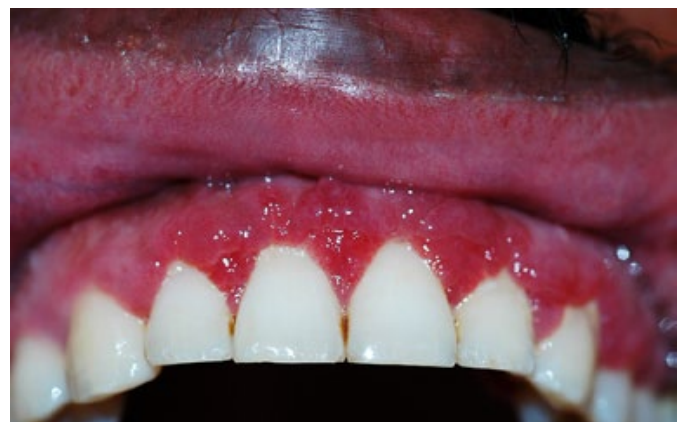

Figure 2. Hyperplastic erythematous lesions on the upper gingival mucosa. 


\section{Answer}

Mucocutaneous leishmaniasis

\section{Discussion}

Polymerase chain reaction disclosed Leishmania spp. The diagnosis of mucocutaneous leishmaniasis (MCL) was made. Intravenous glucantime $(20 \mathrm{mg} / \mathrm{kg} / \mathrm{day})$ for 30 days and pentoxifyline $400 \mathrm{mg}$ three times daily brought to the restitutio ad integrum.

According to the World Health Organization, leishmaniasis is a parasitic protozoal disease transmitted to mammals by phlebotomine sand flies and remains a severe public health problem with 1.5 million new cases a year and a prevalence of 12 million cases worldwide [1-4]. Even though it has long been endemic in developing countries, the economic globalization and the increased volume of international travel have extended its prevalence in developed countries [5].

The Leishmania species are categorized into two groups: Old World (southern Europe, the Middle East, Africa and Asia) and New World leishmaniasis (Latin America; also called American cutaneous leishmaniasis). Old World leishmaniasis is contracted in dry, desert-like climates, while New World leishmaniasis is contracted in jungles, forests, or rural areas. Phlebotomine sand flies transmit the Old World leishmaniasis, while Lutzomyia transmits the New World leishmaniasis [2].

American cutaneous leishmaniasis (ACL) is a zoonotic infection endemic to Latin America. The causative agents include Leishmania (L) (Viannia) (V) braziliensis, L. mexicana, L. (V.) panamensis, and related species. The main reservoirs for L. (V.) braziliensis and other Leishmania (Vianna) spp. are small forest rodents [1-4].

The spectrum of ACL includes single, localized, cutaneous ulcers, diffuse cutaneous leishmaniasis, and mucosal disease (MCL). ACL is most common in persons working at the edge of the forest and among rural settlers. In Brazil, the most frequent etiologic agent of MCL is L. braziliensis, and approximately half of the Brazilian cases are diagnosed in the northern region of the country, including the Brazilian Amazon Basin, a region visited by an increasing number of tourists [1-4].

Infections by L. brasiliensis (but also other species) can "metastasize" to the mucous membranes in about $5 \%$ of the patients suffering from the cutaneous form of ACL. MCL represents the hematogenous or lymphatic dissemination of parasites from the skin to the naso-oropharyngeal mucosa [1-4]. It may develop for months to years after the resolution of the original cutaneous lesion and may well worry tourists who travel to endemic areas [3]. The main clinical feature is a naso-oropharyngeal ulcer, which, if untreated for an extended period of time, can lead to perforation of the septum and even affecting the nose, palate and lips, causing palatal dysfunction, dysphagia, dysphonia, aspiration and severe disfigurement [3].

The differential diagnosis includes mainly deep mycotic infection, rhinoscleroma, Wegener granulomatosis and extranodal NK-T cell lymphoma. Pentavalent antimonials represent the first therapeutic choice; amphotericin B, pentamidine and miltefosine are the main alternatives $[2,3,6]$.

While diagnosis of localized ACL is easy (ulcerated lesions at the site of the bite), MCL may represent a diagnostic pitfall. Delay in the diagnosis may lead to irreversible sequelae. It has recently been shown that the increase in travel to tropical countries has resulted in more cases of imported tropical infections [5]. The incidence of leishmaniasis among travelers returning from endemic areas is 38 per 1,000 patients with cutaneous disorders. This number increases to 143 per 1,000 travelers returning from South America with cutaneous disorders [5]. In the differential diagnosis of nasal ulcers in patients coming from endemic countries, MCL has to be ruled out.

\section{References}

1. Amato VS, Tuon FF, Bacha HA, Neto VA, Nicodemo AC. Mucosal leishmaniasis. Current scenario and prospects for treatment. Acta Trop 2008;105(1):1-9.

2. Schwartz E, Hatz C, Blum J. New world cutaneous leishmaniasis in travellers. Lancet Infect Dis 2006;6(6):342-9.

3. Silveira FT, Lainson R, Corbett CE. Clinical and immunopathological spectrum of American cutaneous leishmaniasis with special reference to the disease in Amazonian Brazil: a review. Mem Inst Oswaldo Cruz 2004;99(3):239-51.

4. Fischer M, GomesPaes M, Reinel D, Talhari S. [Diffuse infiltration of the external ear in a 59-year-old Brazilian patient. "New world" cutaneous leishmaniasis (leishmaniasis tegumentar Americana)]. Hautarzt 2002;53(5):342-6.

5. Freedman DO, Weld LH, Kozarsky PE, et al. Spectrum of disease and relation to place of exposure among ill returned travelers. $\mathrm{N}$ Engl J Med 2006;354(2):119-30.

6. Chrusciak-Talhari A, Dietze R, Chrusciak Talhari C, et al. Randomized controlled clinical trial to access efficacy and safety of miltefosine in the treatment of cutaneous leishmaniasis caused by Leishmania (Viannia) guyanensis in Manaus, Brazil. Am J Trop Med Hyg 2011;84(2):255-60. 